\title{
Densidad de la masa ósea y marcadores bioquímicos del metabolismo óseo: estudio transversal en adultos residentes de Lima Metropolitana, Perú
}

\section{Bone mass density and biochemical markers of bone metabolism: transversal study in adult residents of Metropolitan Lima, Peru}

\author{
${ }^{2}$ Servicio de Endocrinología, Hospital Nacional Dos de Mayo. Lima, Perú. \\ ${ }^{3}$ Servicio de Hematologia, Hospital Nacional Dos de Mayo. Lima, Perú. \\ ${ }^{4}$ Departamento de Medicina, Hospital Nacional Dos de Mayo. Lima, Perú. \\ ${ }^{5}$ Servicio de Reumatología, Hospital Nacional Dos de Mayo. Lima, Perú. \\ ${ }^{6}$ Departamento de Patologia, Hospital Nacional Dos de Mayo. Lima, Perú. \\ ${ }^{7}$ Instituto Nacional Materno Perinatal. Lima, Perú. \\ ${ }^{a}$ Médico endocrinólogo, ORCID: https://orcid.org/ 0000-0002-6513-8743 \\ ${ }^{\mathrm{b}}$ Médica endocrinóloga, ORCID: https://orcid.org/0000-0002-6119-802X \\ 'Médico hematólogo, ORCID: https://orcid.org/0000-0002-7178-9673 \\ ${ }^{d}$ Médica internista, ORCID: htps://orcid.org/0000-0002-6398-2369 \\ ${ }^{e}$ Médica reumatologa, ORCID: https://orcid.org/0000-0003-0386 \\ ${ }^{\dagger}$ Médico reumatólogo, ORCID: https://orcid.org/0000-0002-8354-288 \\ ${ }^{9}$ Técnico en laboratorio, ORCID: https://orcid.org/0000-0001-5375-0102

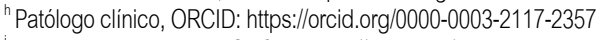 \\ Médica endocrinóloga, ORCID: https://orcid.org/0000-0003-1599-0708 \\ 'Patólogo clínico, ORCID: https://orcid.org/0000-0002-7120-9296 \\ ${ }^{k}$ Médica reumatóloga, ORCID: https://orcid. org/0000-0002-3735-6946+ \\ Médico cirujano, ORCID: https://orcid.org/0000-0002-6086-2505 \\ mMédico cirujano, ORCID: https://orcid.org/0000-0003-3316-4557
}

Fausto Garmendia-Lorenai ${ }^{1, a}$, Rosa Pando-Álvarez ${ }^{1,2, b}$, Oscar Ruiz-Franco, ${ }^{1,3, c}$, Marcia Salas-Pérez ${ }^{1,4, d}$, Ángela ChuquihuaraRodríguez ${ }^{1,5, e}$, Arquímedes Hidalgo-Garcia ${ }^{1,5, f}$, Martín Hernández-Alegre ${ }^{1,6,9}$, Gerardo Ronceros-Medrano ${ }^{1, h}$, Gloria Larrabure-Torrealva ${ }^{1,7, \mathrm{i}}$, José Fuentes-Rivera ${ }^{1, \mathrm{j}}$, Diana Yactayo-Silva ${ }^{1,5, \mathrm{k},}$, Alex Orellana-Cortez ${ }^{1, \mathrm{l}}$, Rafael Pichardo-Rodríguez ${ }^{1,3, \mathrm{~m}}$

${ }^{1}$ Instituto de Investigaciones Clinicas, Facultad de Medicina, Universidad Nacional Mayor de San Marcos. Lima, Perú.

An Fac med. 2020;81(2):148-53. / DOI: https://doi.org/10.15381/anales.v81i2.18106

Correspondencia:

Fausto Garmendia Lorena

fgarmendial@unmsm.edu.pe

Recibido: 13 de marzo 2020

Aceptado: 10 de junio 2020

Publicación en línea: 22 de junio 2020

Conflictos de interés: Los autores declaran no tener conflictos de interés.

Fuente de financiamiento: Fondo concursable 2018, Vicerrectorado de Investigación y Posgrado, Universidad Nacional Mayor de San Marcos.

Citar como: Garmendia-Lorena F, Pando-Álvarez R, Ruiz-Franco $O$, Salas-Pérez M, ChuquihuaraRodríguez A, Hidalgo-García A, et. al. Densidad de la masa ósea y marcadores bioquímicos del metabolismo óseo: estudio transversal en adultos residentes de Lima Metropolitana, Perú. An Fac med. 2020;81(2):148-53. DOl: https:/doi. org/10.15381/anales.v81i2.18106

\section{Resumen}

Objetivos. Determinar y correlacionar la densidad mineral ósea (DMO) y los marcadores bioquímicos del metabolismo óseo de adultos de 30 a 40 años de Lima, Perú, en el año 2018. Métodos. Estudio descriptivo, transversal, realizado a 84 mujeres y 53 varones, de 30 a 40 años de edad, residentes en Lima, Perú, sin manifestaciones ni tratamiento de enfermedades metabólicas óseas primarias ni secundarias. Se registró información del estado actual, antecedentes personales fisiológicos, patológicos, familiares y examen clínico. Se midió la DMO por absorciometría dual de rayos $X$ en columna, cadera y antebrazo; en sangre, parathormona, osteocalcina, 25-hidroxi-vitamina-D, hormona luteinizante, estradiol en las mujeres, testosterona en los varones, calcio sérico, fósforo sérico, fosfatasa alcalina, proteinas totales y fracciones, creatinina; en orina N-telopéptido de enlaces de colágeno tipo I, calcio y fósforo, por métodos convencionales. Resultados. La DMO se efectuó a 62 mujeres y 40 varones; 2 mujeres y 1 varón tuvieron osteoporosis, y 3 mujeres y 2 varones osteopenia, retirados para la evaluación estadistica; 58 mujeres y 35 varones tuvieron una DMO normal. Los varones tuvieron mayor DMO y concentraciones mayores de calcio, fosfatasa alcalina y creatinina; 14 mujeres y 2 varones tuvieron cifras bajas de 25-OH-vitaminaD. Hubo correlación positiva entre DMO y el IMC. Conclusiones. La DMO normal de personas de 30 a 40 años de Lima fue lineal y hubo una relación positiva con el IMC; hubo osteoporosis en 2 mujeres y 1 varón, osteopenia en 3 mujeres y 2 varones, cifras bajas de 25-hidroxi-vitamina-D en 16 personas.

Palabras clave: Densidad Ósea; Biomarcadores; Metabolismo; Adultos; Perú (fuente: DeCS BIREME).

\section{Abstract}

Objectives. To determine and correlate the bone mineral density (BMD) and the biochemical markers of bone metabolism in adults 30 to 40 years old from Lima, Perú, in year 2018. Methods. A total of 84 women and 53 men, 30 to 40 years old from Lima, Perú, were submmited to a descriptive, transversal study. They did not have symptoms nor treatment for bone metabolic diseases. Information about their present health state, personal and familiar physiological and pathological history and physical examinatión were registered. BMD was measured by dual energy-X-ray absorptiometry in spine, hip and forearm. Parathormone, osteocalcine, 25-hidroxi-vitamine-D, luteinizing hormone, estradiol in women, testosterone in men, calcium, phosphorus, alkaline fosfatase, total and fractionated proteins, creatinine were measured in blood; in urine N-telopéptide, calcium and phosphorus by conventional methods. Results. BMD was measured in 62 women and 40 males; 2 women and 1 man had osteoporosis and 3 women and 2 men osteopenia, who were taken out of statistical evaluation; 58 women and 35 men showed a normal BMD. Male subjects had a greater $\mathrm{BMD}$, higher calcium, alkaline fosfatase and creatine blood concentrations than the female ones; 14 women y 2 men had low $25-\mathrm{OH}-$ vitaminaD concentrations. A positive correlation between the BMD and BMI was found. Conclusions. The normal BMD of persons 30 to 40 years old showed a linear figure and there was a positive correlate with the BMl; premature osteoporosis in 2 women and 1 men as well as osteopenia in 3 women y 2 men was found, low concentrations of 25-hidroxi-vitamine-D was detected in 16 persons. Keywords: Bone Density; Biomarkers; Metabolism; Adult; Peru (source: MeSH NLM). 


\section{INTRODUCCIÓN}

El sistema esquelético no solo tiene funciones de sostén, locomoción, protección de órganos vitales y hematopoyesis, sino que además posee un metabolismo mineral y proteico activo y permanente, que varía con la edad, sexo, actividad física, nutrición, gestaciones, lactancia, menopausia y constitución genética; en cuya regulación participan factores tanto sistémicos como locales ${ }^{(1,2)}$.

El metabolismo óseo se encuentra en un permanente recambio de formación y resorción, en él intervienen factores locales como sistémicos; los factores locales están representados por los osteoblastos y osteoclastos, y los sistémicos por los marcadores bioquímicos parathormona, calcitonina, hormonas sexuales, esteroides suprarrenales, insulina, hormonas tiroideas, somatotropina, vitamina D, calcio y fósforo. Se ha demostrado que existe una disminución gradual de los marcadores bioquímicos con la edad, que se los suele medir en una forma simplificada para la formación ósea, por las concentraciones sanguíneas de calcitonina y fosfatasa alcalina y la resorción ósea por Ntelopéptido de enlaces de colágeno tipo I (NTX) en orina ${ }^{(3)}$.

En el metabolismo óseo normal, la cantidad de hueso o masa ósea comprende, primero a un proceso de formación que, por lo general, dura desde la niñez hasta los 25 a 30 años, edad en la que llega a su máximo desarrollo, denominado pico de masa ósea (PMO) ${ }^{(4,5)}$, que se mantiene igual por algunos años, y luego se observa una disminución gradual de la masa ósea vinculada al proceso de envejecimiento ${ }^{(6)}$ y más rápida en las mujeres a partir de la menopausia(6). El proceso de formación es de vital importancia. Si ocurre una deficiente formación, existe mayor probabilidad de desarrollar deficiencia de masa ósea y osteoporosis, condiciones que facilitan la producción de fracturas patológicas de cadera, columna vertebral y/o antebrazo aún en adultos jóvenes ${ }^{(6,7)}$.

En el presente estudio se aporta conocimiento sobre la fisiología y patología del metabolismo óseo de un segmento poblacional representativo importante del Perú. La población de Lima Metropolitana constituye un tercio de la población del Perú, que en el año 2018 alcanzó a 31237400 habitantes ${ }^{(8)}$, ubicado a nivel del mar, en la gran mayoría de raza mestiza, mezcla de la población amerindia con la hispana, que se encuentra en una edad, en la que se ha alcanzado el pico de masa ósea y antes de la normal declinación por la edad y la menopausia, al mismo tiempo que se proporciona información adicional de correlación con los marcadores bioquímicos del metabolismo óseo

\section{MÉTODOS}

\section{Diseño del estudio}

Se llevó a cabo un estudio descriptivo, transversal, en adultos residentes de Lima Metropolitana durante el año 2018, en el Instituto de Investigaciones Clínicas de la Facultad de Medicina de la Universidad Nacional Mayor de San Marcos.

\section{Población y muestra}

Participaron del estudio personas de ambos sexos, comprendidos entre los 30 a 40 años, que residían en Lima Metropolitana y aceptaron voluntariamente participar en el estudio con la firma del consentimiento informado. Se excluyeron a aquellos que presentaron el diagnóstico o estaban en tratamiento de alguna enfermedad metabólica de hueso, uso crónico de corticosteroides, hipogonadismo, fracturas patológicas, alteraciones significativas del ciclo menstrual y otras causas de osteoporosis secundaria.

Basado en el nivel de confianza del 95\%, una precisión de 5, para una población finita con una varianza de 26 , agregándose un porcentaje de pérdidas del $15 \%$, se requirió un total de 122 adultos, de acuerdo a la fórmula de población infinita: $n=\left(\frac{z_{\alpha / 2} \alpha x}{d}\right)^{2}$. Donde $Z \alpha / 2=1,96, \sigma: 26, d: 5$.

Posteriormente, la selección de los participantes fue de manera no probabilística mediante un muestreo consecutivo.

\section{Variables de estudio}

La medición de la densitometría ósea (DMO) se efectuó por absorciometría dual de rayos $X$ (DEX) con un equipo Ho- logic ${ }^{\circledR}$ en la columna lumbar (Col), cadera total (CadT), cadera derecha (CadD), cadera izquierda (Cadl) y antebrazo (Ant), cuyos resultados se evaluaron de acuerdo a los criterios de la OMS ${ }^{(9)}$ y la International Society for Clinical Densitometry (ISCD) ${ }^{(10)}$, que se expresó tanto en g/ $\mathrm{cm}^{2}$, así como en el score $T$ y el score $Z$. La DMO se consideró normal cuando el score $T$ fue $\geq$ de-1,5/DS en columna y/o cadera; osteopenia cuando el score estuvo entre-1,5 y-2,5/DS; y osteoporosis cuando fue inferior a -2,50/DS. La DMO se realizó en la institución IMPPARES.

Adicionalmente, en una muestra de sangre venosa periférica en ayunas de los participantes del estudio, se determinó la parathormona (PTH), osteocalcina (OC), 25-hidroxi-vitamina D (25-OH-D), hormona luteinizante $(\mathrm{LH})$, en mujeres estradiol (E2), en varones testosterona ( $T$ ), calcio sérico (Cas), fósforo sérico (Ps), fosfatasa alcalina (FA), proteínas totales y fraccionadas, creatinina; y en orina N-telopéptido de enlaces de colágeno tipo I (NTX), calcio (Cao) y fósforo (Po), por métodos convencionales (AT Biotec).

Se recopiló la información necesaria en una ficha confeccionada en base a los objetivos del estudio, consignando los datos de filiación, sintomatología actual, posible medicación, antecedentes personales fisiológicos y patológicos, antecedentes familiares. Asimismo, se tomaron las medidas antropométricas, presión arterial y examen físico, a los participantes del estudio.

\section{Análisis estadístico}

Se presentan medidas de resumen en frecuencias y porcentajes para las variables cualitativas, así como medias y desviación estándar para las variables cuantitativas. Para el análisis bivariado se utilizó la prueba T para dos muestras independientes. Posteriormente, para la evaluación de la diferencia entre diversas categorías, se requirió la aplicación de la prueba ANOVA de un factor. Se consideró estadisticamente significativo a todo valor de $p<0,05$. Los datos fueron procesados en el software estadístico SPSS versión 23.

\section{Aspectos éticos}

La investigación tuvo la aprobación del Comité de Ética del Hospital Nacional Dos de Mayo. 


\section{RESULTADOS}

De un total de 137 participantes, solo 102 cumplieron con los criterios de selección y se les efectuó la densitometría ósea (62 mujeres y 40 varones), de los cuales se retiraron para los cálculos estadísticos a 2 mujeres y 1 varón por tener osteoporosis, 3 mujeres y 2 varones por tener osteopenia. Las características generales de 93 personas normales, 58 mujeres premeno- páusicas y 35 varones con DMO normal, se encuentran en la tabla 1.

Los varones tuvieron el peso, talla y circunferencia abdominal (CA) mayores que las mujeres; pero el índice de masa corporal (IMC) fue semejante; 24 mujeres y 6 varones tenían un IMC normal, 25 mujeres y 22 varones tenían sobrepeso y 9 mujeres y 7 varones eran obesos (Tabla 2). Todos los participantes del estu-

Tabla 1. Características generales de la muestra ( $n=93)$. Participantes residentes en Lima Metropolitana, en quienes se determinó la densidad de masa ósea y marcadores bioquímicos de metabolismo óseo.

\begin{tabular}{|c|c|c|c|}
\hline & Mujeres & Varones & $\mathbf{p}$ \\
\hline $\mathrm{N}$ & 58 & 35 & \\
\hline Edad, años & $34,8 \pm 3,22$ & $35,0 \pm 3,58$ & 0,739 \\
\hline Peso, kg & $65,0 \pm 10,2$ & $81,5 \pm 14,1$ & 0,000 \\
\hline Talla, m & $1,56 \pm 0,06$ & $1,70 \pm 0,06$ & 0,000 \\
\hline IMC, peso/talla² & $26,5 \pm 3,73$ & $27,9 \pm 4,38$ & 0,115 \\
\hline $\mathrm{CA}, \mathrm{cm}$ & $87,5 \pm 9,48$ & $97,5 \pm 9,97$ & 0,000 \\
\hline PAs, $\mathrm{mmHg}$ & $104,7 \pm 11,0$ & $110,8 \pm 13,1$ & 0,025 \\
\hline PAd, $\mathrm{mmHg}$ & $69,1 \pm 8,17$ & $71,6 \pm 8,32$ & 0,169 \\
\hline Cas, mg/dL & $9,39 \pm 1,50$ & $10,0 \pm 0,84$ & 0,009 \\
\hline $\mathrm{Ps}, \mathrm{mg} / \mathrm{dL}$ & $4,78 \pm 0,92$ & $4,67 \pm 0,83$ & 0,605 \\
\hline PTH, pg/mL & $24,2 \pm 8,87$ & $25,1 \pm 9,83$ & 0,666 \\
\hline $\mathrm{OC}, \mathrm{ng} / \mathrm{mL}$ & $9,61 \pm 3,38$ & $10,3 \pm 5,07$ & 0,357 \\
\hline VitD, ng/mL & $36,9 \pm 8,58$ & $41,3 \pm 8,26$ & 0,030 \\
\hline FA, UI/L & $168,5 \pm 49,3$ & $193,5 \pm 56,4$ & 0,042 \\
\hline PT, g/dL & $7,44 \pm 0,51$ & $7,81 \pm 0,58$ & 0,004 \\
\hline $\mathrm{ALB}, \mathrm{g} / \mathrm{dL}$ & $4,08 \pm 0,28$ & $4,11 \pm 0,45$ & 0,735 \\
\hline GLOB g/dL & $3,37 \pm 0,49$ & $3,69 \pm 0,61$ & 0,015 \\
\hline $\mathrm{Cr}, \mathrm{mg} / \mathrm{dL}$ & $0,85 \pm 0,10$ & $1,02 \pm 0,15$ & 0,000 \\
\hline NTX, nMECO/mM Cr & $70,9 \pm 42,9$ & $59,5 \pm 32,7$ & 0,215 \\
\hline Cao, mg & $17,7 \pm 7,32$ & $16,6 \pm 6,16$ & 0,501 \\
\hline Po, mg & $22,3 \pm 16,5$ & $22,6 \pm 11,8$ & 0,930 \\
\hline $\mathrm{LH}, \mathrm{mul} / \mathrm{Ml}$ & $6,16 \pm 4,50$ & $3,23 \pm 2,06$ & 0,000 \\
\hline E2, & $85,1 \pm 72,3$ & & \\
\hline $\mathrm{T}$ & & $3,99 \pm 1,22$ & \\
\hline DMOcol, g/cm², & $1,030 \pm 0,09$ & $1,090 \pm 0,12$ & 0,016 \\
\hline DMOcadT, g/cm² & $0,998 \pm 0,10$ & $1,082 \pm 0,12$ & 0,002 \\
\hline DcadD, g/cm², & $0,845 \pm 0,10$ & $0,940 \pm 0,13$ & 0,001 \\
\hline Dcadl, , g/cm², & $0,840 \pm 0,10$ & $0,943 \pm 0,12$ & 0,000 \\
\hline Dant, g/cm², & $0,598 \pm 0,07$ & $0,694 \pm 0,08$ & 0,000 \\
\hline
\end{tabular}

IMC: índice de massa corporal; CA: circunferência abdominal; PAs: presión arterial sistólica; PAd: presión arterial diastólica; Cas: cálcio sérico; Os: fósforo sérico; PTH: parathormona; OC: osteocalcina; VitD: vitamina D; FA: fosfatasa alcalina; PT: proteínas totales; ALB: albumina; GLOB: globulinas; $\mathrm{Cr}$ : creatinina; NTX: N-telopéptido de enlaces de colágeno tipo I; Cao: calcio urinario; Po: fósforo urinario; LH: hormona luteinizante; E2: estradiol; T: testosterona; DMOcol: DMO de columna; DMOcadT: DMO de cadera total; DcaDd: DMO de cadera derecha; DMOI: DMO de cadera izquierda; DMOant: DMO de antebrazo.

dio tuvieron presión arterial normal, los varones tuvieron una PAs mayor que las mujeres. Los varones tuvieron concentraciones mayores de Cas, 25-OH-D, FA, PT, Glob y creatinina en sangre; 14 mujeres y 2 varones tuvieron cifras bajas de $25-\mathrm{OH}$ D (rango normal $30-100 \mathrm{mg} / \mathrm{dL}$ ), cuyas DMO no fueron diferentes a las personas con cifras normales.

Las concentraciones de LH y E2 estuvieron dentro del rango normal de acuerdo a las etapas del ciclo menstrual de las mujeres; las concentraciones de $\mathrm{LH}$ y $\mathrm{T}$ dentro de lo normal en los varones. El promedio de la DMO fue mayor en los varones en todos los segmentos corporales examinados (Tabla 3 y Figura 1). Los marcadores bioquímicos de función renal y hepática estuvieron dentro de límites normales. Hubo correlación positiva entre DMO y el IMC (Tablas 4 y 5).

\section{DISCUSIÓN}

En este trabajo, se da a conocer, por primera vez, las características de la densidad de masa ósea, al mismo tiempo que los marcadores bioquímicos del metabolismo óseo, de una muestra de una población de adultos jóvenes normales comprendidos entre los 30 a 40 años de edad, de ambos sexos, residentes en Lima Metropolitana, Perú. En ese rango de edad no hubo diferencias en la densidad de la masa ósea, se entiende luego de haber alcanzado el pico de masa ósea.

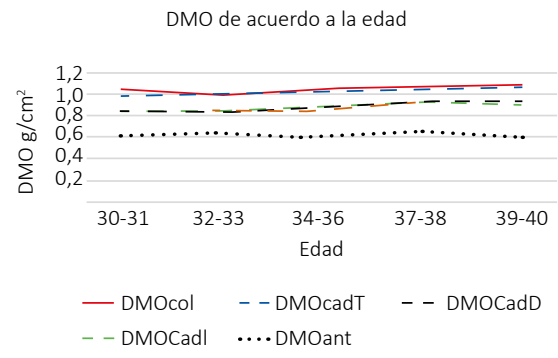

Figura 1. Densitometría ósea de acuerdo a la edad, en residentes de Lima Metropolitana ( $n=102$ ), en quienes se determinó la densidad de masa ósea y marcadores bioquímicos de metabolismo óseo.

DMO col: DMO en columna

DMOcadT: DMO en cadera total

DMO CadD: DM en cadera derecha

DMO Cadl: DMO en cadera izquierda

DMO ant: DMO en antebrazo 
Tabla 2. Valores $p$ de las correlaciones bivariadas positivas entre densidad mineral ósea y marcadores bioquímicos de metabolismo óseo ( $\mathrm{n}=102$ ).

\begin{tabular}{lcccccccccccccc} 
& Edad & IMC & CA & PAs & PAd & Cas & Ps & PTH & OC & 25OHD & LH & E2 & T \\
DMOcol & 0,034 & 0,000 & 0,000 & 0,001 & 0,033 & 0,855 & 0,403 & 0,221 & 0,212 & 0,043 & 0,129 &, 040 & 0,912 \\
\hline T & 0,027 & 0,000 & 0,000 & 0,006 & 0,073 & 0,705 & 0,568 & 0,433 & 0,372 & 0,211 & 0,088 &, 064 & 0,573 \\
\hline Z & 0,007 & 0,000 & 0,000 & 0,015 & 0,172 & 0,972 & 0,407 & 0,476 & 0,439 & 0,236 & 0,083 &, 069 & 0,406 \\
\hline DMOcadT & 0,001 & 0,000 & 0,000 & 0,027 & 0,349 & 0,510 & 0,360 & 0,078 & 0,071 & 0,455 & 0,054 &, 554 & 0,990 \\
\hline T & 0,017 & 0,013 & 0,001 & 0,885 & 0,282 & 0,486 & 0,986 & 0,000 & 0,000 & 0,027 & 0,199 &, 678 & 0,607 \\
\hline Z & 0,038 & 0,035 & 0,001 & 0,663 & 0,178 & 0,586 & 0,736 & 0,000 & 0,000 & 0,013 & 0,141 &, 885 & 0,427 \\
\hline DMOcadD & 0,020 & 0,002 & 0,000 & 0,295 & 0,875 & 0,361 & 0,286 & 0,232 & 0,210 & 0,515 & 0,140 &, 690 & 0,836 \\
\hline T & 0,045 & 0,002 & 0,261 & 0,208 & 0,917 & 0,384 & 0,548 & 0,017 & 0,010 & 0,235 & 0,870 &, 995 & 0,640 \\
\hline Z & 0,011 & 0,001 & 0,214 & 0,471 & 0,643 & 0,469 & 0,796 & 0,027 & 0,019 & 0,272 & 0,476 &, 684 & 0,362 \\
\hline DMOcadl & 0,010 & 0,002 & 0,001 & 0,068 & 0,586 & 0,281 & 0,578 & 0,248 & 0,253 & 0,544 & 0,057 &, 776 & 0,978 \\
\hline T & 0,002 & 0,001 & 0,025 & 0,170 & 0,607 & 0,954 & 0,593 & 0,022 & 0,023 & 0,292 & 0,671, &, 889 & 0,453 \\
\hline Z & 0,004 & 0,000 & 0,000 & 0,046 & 0,364 & 0,706 & 0,679 & 0,038 & 0,039 & 0,332 & 0,357 &, 974 & 0,337 \\
\hline DMOant & 0,330 & 0,004 & 0,000 & 0,206 & 0,825 & 0,045 & 0,009 & 0,180 & 0,199 & 0,617 & 0,247 &, 223 & 0,526 \\
\hline T & 0,379 & 0,448 & 0,421 & 0,185 & 0,136 & 0,922 & 0,222 & 0,003 & 0,003 & 0,211 & 0,020 &, 523 & 0,415 \\
\hline Z & 0,102 & 0,217 & 0,729 & 0,386 & 0,065 & 0,748 & 0,341 & 0,002 & 0,002 & 0,126 & 0,031 &, 861 & 0,770 \\
\hline
\end{tabular}

IMC: índice de massa corporal; CA: circunferência abdominal; PAs: presión arterial sistólica; PAd: presión arterial diastólica; Cas: cálcio sérico; PTH: parathormona; OC: osteocalcina; 25ODH: vitamina D; LH: hormona luteinizante; E2: estradiol; T: testosterona.

DMO col: DMO en columna

DMOcadT: DMO en cadera total

DMO CadD: DM en cadera derecha

DMO Cadl: DMO en cadera izquierda

DMO ant: DMO en antebrazo

Se ha constatado una relación positiva entre la DMO y el IMC; así, las persona con sobrepeso y obesidad tuvieron una DMO mayor que aquellas con peso normal tanto en la columna como en la cadera total, de carácter significativo en las regiones del cuerpo que sostienen el mayor peso corporal, es decir la columna vertebral y la caderal ${ }^{(11,12)}$. Maceda y col. comprobaron que a tanto mayor el IMC, menor fue la prevalencia de osteoporosis en personas mayores de 50 años ${ }^{(13)}$. Un aspecto que requiere un análisis especial es la existencia de osteoporosis en personas premenopáusicas y en varones jóvenes (14,15); en nuestro caso hemos encontrado $8,6 \%$ de personas que tenía osteoporosis u osteopenia antes de los 40 años, que se suele atribuir a causas secundarias y a factores que interfieren en una apropiada formación ósea hasta alcanzar el pico de masa ósea ${ }^{(14)}$. Los factores que intervienen en este proceso son múltiples, varios de los cuales hemos descartado como el uso prolongado de glucocorticoides, hipogonadismo, hiperparatiroidis- mo, osteomalacia, alteraciones renales, alteraciones hepáticas, FA normal OC, NXT; por el contrario, no se puede descartar totalmente el IMC debajo de lo normal en 1 caso. Además, podría ser un factor el hecho que la intolerancia a la lactosa, condición importante en el Perú, cuya aparición y duración es muy variable para apreciar una menor ingesta de leche como fuente de calcio ${ }^{(16)}$. La actividad física tampoco es fácil de cuantificar, más aun cuando se ha demostrado que el tipo de actividad tiene una diferente

Tabla 3. Valores p según ANOVA de un factor, entre densidad mineral ósea y marcadores bioquímicos de metabolismo óseo (n=102).

\begin{tabular}{lccccccccccc} 
& Edad & Peso & IMC & Cas & PAs & PTH & PT & ALB & GLOB \\
DMOcol & 0,485 & 0,419 & 0,053 & 0,073 & 0,364 & 0,020 & 0,742 & 0,163 & 0,026 \\
\hline DMOcadT & 0,120 & 0,039 & 0,006 & 0,007 & 0,164 & 0,336 & 0,089 & 0,018 & 0,141 \\
\hline DMOcadD & 0,641 & 0,030 & 0,131 & 0,074 & 0,007 & 0,752 & 0,012 & 0,014 & 0,227 \\
\hline DMOcadl & 0,135 & 0,099 & 0,056 & 0,063 & 0,075 & 0,399 & 0,010 & 0,018 & 0,441 \\
\hline DMOant & 0,178 & 0,341 & 0,303 & 0,088 & 0,308 & 0,524 & 0,928 & 0,668 & 0,176 \\
\hline
\end{tabular}

IMC: índice de massa corporal; Cas: cálcio sérico; PAs: presión arterial sistólica; PTH: parathormona; PT: proteínas totales; ALB: albumina; GLOB: globulinas.

DMO col: DMO en columna

DMOcadT: DMO en cadera total

DMO CadD: DM en cadera derecha

DMO Cadl: DMO en cadera izquierda

DMO ant: DMO en antebrazo 
Tabla 4. Densidad mineral ósea y marcadores bioquímicos de metabolismo óseo en los participantes del estudio con diagnóstico de osteoporosis y osteopenia ( $\mathrm{n}=8$ ).

\begin{tabular}{|c|c|c|c|c|c|c|c|c|c|c|c|c|c|c|c|c|c|c|c|}
\hline \multirow[t]{2}{*}{ Nombre } & \multirow{2}{*}{$\begin{array}{l}\text { Edad } \\
\text { Sexo }\end{array}$} & \multicolumn{3}{|c|}{ Columna } & \multicolumn{3}{|c|}{ Cadera total } & \multicolumn{3}{|c|}{ Cadera derecha } & \multicolumn{3}{|c|}{ Cadera Izquierda } & \multirow[t]{2}{*}{ CAS } & \multirow[t]{2}{*}{ Ps } & \multirow[t]{2}{*}{ PTH } & \multirow[t]{2}{*}{ oc } & \multirow[t]{2}{*}{ 250HD } & \multirow[t]{2}{*}{ NTX } \\
\hline & & DMO & $T$ & Z & DMO & $T$ & z & DMO & $T$ & $\mathbf{z}$ & DMO & $T$ & z & & & & & & \\
\hline EDL & 39 우 & 0,737 & $-2,8$ & $-2,6$ & 0,814 & $-1,1$ & $-1,1$ & 0,722 & $-1,3$ & $-1,2$ & 0,692 & $-1,5$ & $-1,5$ & 8,48 & 5,77 & 17,60 & 9,87 & 35,70 & 55,40 \\
\hline ACR & 33 운 & 0,726 & $-2,9$ & $-2,9$ & 0,784 & $-1,3$ & $-1,3$ & 0,702 & $-1,5$ & $-1,5$ & 0,671 & $-1,7$ & $-1,7$ & 10,06 & 5,16 & 28,59 & 12,99 & 43,61 & \\
\hline PHP & $30 \pi$ & 0,811 & $-2,5$ & $-2,5$ & 0,833 & $-1,3$ & $-1,2$ & 0,759 & $-1,7$ & $-1,4$ & 0,701 & $-2,1$ & $-1,8$ & 9,04 & 3,97 & 26,82 & 15,42 & 34,00 & \\
\hline SLLF & $30 q$ & 0,870 & $-1,6$ & $-1,6$ & 1,014 & 0,4 & 0,4 & 0,843 & $-0,3$ & $-0,2$ & 0,850 & $-0,2$ & $-0,2$ & 8,80 & 7,52 & 16,28 & 10,82 & 30,79 & 11,18 \\
\hline PKC & $380^{\pi}$ & 0,894 & $-1,8$ & $-1,7$ & 0,984 & $-0,5$ & $-0,3$ & 0,781 & $-1,5$ & $-0,9$ & 0,769 & $-1,6$ & -1 & 9,20 & 2,70 & 35,24 & & 41,33 & \\
\hline GMM & $330^{1}$ & 0,911 & $-1,6$ & $-1,6$ & 0,854 & $-1,5$ & $-1,3$ & 0,827 & $-1,1$ & $-0,7$ & 0,777 & $-1,5$ & $-1,1$ & 10,17 & 4,20 & 17,48 & 19,80 & 55,93 & 11,05 \\
\hline ECR & 32 웅 & 0,859 & $-1,7$ & $-1,7$ & 0,784 & $-1,3$ & $-1,3$ & 0,697 & $-1,5$ & $-1,5$ & 0,695 & $-1,5$ & $-1,5$ & 10,06 & 5,07 & 36,26 & 14,50 & 26,27 & 39,06 \\
\hline ORO & $35 \pi$ & 0,855 & $-2,1$ & $-2,1$ & 0,939 & $-0,9$ & $-0,6$ & 0,725 & $-1,9$ & $-1,4$ & 0,769 & $-1,6$ & $-1,1$ & 10,16 & 5,31 & 19,24 & 8,90 & 43,90 & 12,69 \\
\hline
\end{tabular}

CAS: cálcio sérico; Ps: presión arterial sistólica; PTH: parathormona; OC: osteocalcina; 25ODH: vitamina D; NTX: N-telopéptido de enlaces de colágeno tipo I

influencia sobre la masa ósea; así, las atletas de competencia suelen desarrollar la tríada de trastornos menstruales, menor disponibilidad de energía y menor densidad ósea. Todo lo cual nos inclina a pensar que los casos hallados constituirían formas genéticas.

Se ha demostrado que existen diferencias raciales en la adquisición de la masa ósea. Bachrack y col. encontraron que las mujeres de raza negra tienen cifras más altas de DMO que las no negras y que no hubo diferencias significativas entre blancas, hispánicas y asiáticas; del mismo modo los varones de raza negra tuvieron cifras mayores de DMO que los de otras razas, pero los blancos y asiáticos tuvieron mayor DMO que los hispánicos ${ }^{(17)}$. La diferencia racial también ha sido reporta-

da en personas normales y con afección renal por Jorletti y col. ${ }^{(18)}$. La mayoría de la población peruana es mestiza, entre hispánica y nativa. La población de Lima Metropolitana comprende a un tercio de la población peruana ${ }^{(8)}$, por lo que cobra singular significado establecer los patrones de normalidad de esta población, cuyo rastreo genómico demuestra que en el mestizaje existente, el componente europeo es pequeño pero significativo ${ }^{(19)}$. En la población mestiza de México, se ha encontrado que existen diferencias en la DMO entre las poblaciones del norte, centro y sur del país (20); esta realidad también es marcada en el Perú, que posee regiones tan dispares como la costa, sierra y selva, tanto en lo que se refiere a la altitud, presión barométrica, actividad física, como de los rastros genómicos ${ }^{(19)}$.

Tabla 5. Resultado de la estimación ANOVA para el factor IMC en los participantes del estudio, residentes en Lima Metropolitana, en quienes se determinó la densidad de masa ósea y marcadores bioquímicos de metabolismo óseo $(n=93)$.

\begin{tabular}{lcccc} 
Variable & Normal & Sobrepeso & Obesidad & Anova $\mathbf{p}$ \\
$\mathrm{n}$ & 30 & 47 & 16 & \\
\hline IMC & 23,2 & 27,2 & 33,7 & \\
\hline DMOCol & 1,007 & 1,053 & 1,138 & 0,022 \\
\hline DMOCadT & 0,98 & 1,03 & 1,122 & 0,009 \\
\hline DMOCadD & 0,845 & 0,881 & 0,946 & 0,142 \\
\hline DMOCadl & 0,836 & 0,88 & 0,953 & 0,077 \\
\hline DMPant & 0,593 & 0,645 & 0,679 & 0,194 \\
\hline CaS & 9,49 & 9,75 & 9,59 & 0,998 \\
\hline PS & 4,84 & 4,65 & 4,8 & 0,452 \\
\hline PTH & 23,9 & 24,6 & 25,5 & 0,328 \\
\hline $25-O H-D$ & 35,5 & 41 & 37,2 & 0,262 \\
\hline LH & 6,22 & 5,34 & 4,13 & 0,127 \\
\hline E2 & 70,2 & 106,9 & 68,6 & 0,806 \\
\hline T & 4,25 & 4,14 & 3,37 & 0,738 \\
\hline
\end{tabular}


marcadores bioquímicos que intervienen en el metabolismo óseo como la hormona de crecimiento, insulina, hormonas tiroideas; así como en el hecho que el número de varones incorporados fue menor que el de mujeres, que en la edad de 36 años se contó solo con 2 mujeres, y que la muestra fue no probalística.

Se concluye, que en 58 mujeres y 35 varones normales, de 30 a 40 años de edad, de Lima Metropolitana se ha establecido la densidad mineral ósea, normal de carácter uniforme durante los 11 años de estudio, que revela que se ha alcanzado el pico de masa ósea y que durante ese lapso existe un plateau sin variación significativa. El promedio de la DMO fue mayor en los varones que en las mujeres en todos los segmentos corporales estudiados y tuvo una correlación positiva con el IMC. Un hallazgo adicional es la existencia de osteoporosis en 2 mujeres y 1 varón, y osteopenia en 3 mujeres y 2 varones, que estarían en relación a factores genéticos, dado que no se encontraron factores que influenciaran la pérdida de masa ósea tales como el uso crónico de corticoides, alcoholismo, inmovilización prolongada, hipogonadismo, trastornos importantes del ciclo menstrual, uso de anticonceptivos progestacionales de efecto prolongado, que sustenten el origen de esta deficiencia metabólica ósea ${ }^{(13,14)}$.

La determinación de los marcadores bioquímicos del metabolismo óseo, al mismo tiempo que la medición de la densitometría ósea, es fundamental para efectuar el diagnóstico diferencial con otras enfermedades metabólicas óseas como se hay podido efectuar en el presente trabajo.

La realización de esta investigación en personas de 30 a 40 años permite conocer a que edad se efectúa el píco de masa ósea en la población estudiada y los resultados obtenidos son básicos para estudios posteriores, en los que se tiene previsto estudiar las características del metabolismo óseo de la población general peruana, que permita conocer el patrón de normalidad, así como la prevalencia de patología del metabolismo óseo en su gran diversidad ambiental y bioétnica ${ }^{(19)}$.

\section{REFERENCIAS BIBLIOGRÁFICAS}

1. Garmendia F. Metabolismo normal y patológico del hueso. Diagnóstico. 2011;50(1):11-13.

2. Walsh JS. Normal bone physiology, remodelling and its hormonal regulation. Surgery. 2017; 36 (1): 1-6. DOI: https://doi.org/10.1016/j.mpsur.2017.10.006

3. Szulc P, Garnero P, Munoz F, Marchand F, Delma DP. Cross-sectional Evaluation of Bone Metabolism in Men. J Bone Mineral Reseach. 2001; 16 (9): 1642-1650. DOI: DOI: 10.1359/ jbmr.2001.16.9.1642

4. Bonjour JP, Theintz G, Buchs B, Slosman D, Rizzoli R. Critical years and stages of puberty for spinal and femoral bone mass accumulation during adolescence. J Clin Endocrinol Metab 1991;73:555-63. DOI: 10.1210/jcem-73-3-555

5. Baxter-Jones ADG, Faulkner RA, Forwood MR, Mirwald RL, Bailey DA. Bone mineral accrual from 8 to 30 years of age: An Estimation of Peak Bone Mass. JBMR. 2011; 26 (8): 1729-1739. DOI: 10.1002/jbmr.412

6. Demontiero O, Vidal C, Duque. Aging and bone loss: new insights for the clinician. Ther Adv Musculoskel Dis. 2012; 4(2) 61-76. DOI: 10.1177/ $1759720 \times 11430858$

7. Daly RM, Rosengren BE, Alwis G, Ahlborg HG, Sernbo I, Karlsson MK. Gender specific age-related changes in bone density, muscle strength and functional performance in the elderly: a-10 year prospective population-based study. BMC Geriatrics. 2013;13:71. DOI: 10.1186/1471-2318-13-71

8. Institutito Nacional de Estadística e Informática [Internet]. Nota de Prensa, 25 de junio 2018 [Fecha de acceso: 8 de marzo 2020]. Disponible en: https://www.inei.gob.pe/media/MenuRecursivo/ noticias/nota-de-prensa-no-108-2018-inei.pdf. 25 de junio 2018

9. World Health Organization [Internet]. Assesment of fracture risk and its application to screening for postmenopausal osteoporosis: report of a WHO study group [Fecha de acceso: 8 de marzo 2020]. Disponible en: https://apps.who.int/iris/ handle/10665/39142

10. International Society for Clinical Densitometry [Internet]. Official Position Adults, 2019 [Fecha de acceso: 8 de marzo 2020]. Disponible en: https:// www.iscd.org/official-positions/2019-iscd-officialpositions-adult/

11. Felson DT, Zhang $\mathrm{Y}$, Hannan MT, Anderson JJ. Effects of weight and body mass index on bone mineral density in men and women: The Framingham study. 1993; 8 (5): 567-573. DOI: https:// doi.org/10.1002/jbmr.5650080507

12. Benner A, Hammoudeii M, Zirie M, Heller RF. Is the obesity a protective factor for osteoporosis? Aplar Journal of Reumatology. 2005; 8: 32-38. DOI: https://doi.org/10.1111/j.1479-8077.2005.00119.x

13. Maceda WA, Maceda DP, Maceda M, martinez R, Valcárcel LF. Disminución mineralósea en pacientes mayores de 50 años y su relación con el IMC. Diagnóstico 2011; 50 (3): 123-31.
14. Cohen A. Premenopausal osteoporosis. Endocrinol Metab Clin N Am. 2017; 46 (1): 117-133. DOI: 10.1016/j.ecl.2016.09.007

15. Ferrari S, Bianchi ML, Eisman JÁ, Foldes AJ, Adami $\mathrm{S}$, Wahl DA, et al. Osteoporosis in young adults: pathophysiology, diagnosis and management. Osteoporosis Int. 2012; 23: 2735-48. DOI: 10.1007/ s00198-012-2030-x

16. Figueroa R, Melgar E, Jo N, Garcia O. Intestinal lactase deficiency in an apparently normal Peruvian population. Am J Digest Dis. 1971;16 (10): 881-889.

17. Bachrach LK, Hastie T, Wang MC, Narasimham B, Markus R. Bone Mineral Acquisition in Healthy Asian, Hispanic, Black, and Caucasian Youth: A Longitudinal Study. J Clin Endocrinol Metab. 1999; 84 (12): 4702-2. DOI: https://doi.org/10.1210/ jcem.84.12.6182.

18. Jorgetti V, dos Reis, Ott SM. Ethnic differences in bone and mineral metabolism in healthy people and patients with CKD. Kidney International. 2013; 85,1283-1289. DOI: doi:10.1038/ki.2013.443

19. Sandoval JR, Salazar-Granara A, Acosta O, Castillo-Herrera W, Fujita R, Pena SDJ, et al. Tracing the genomic ancestry of Peruvians reveals a major legacy of pre-Columbian ancestors. Journal of Human Genetics. 2013;58(9): 627-34. DOI: DOI: 10.1038/jhg.2013.73

20. Tamayo J, Diaz R, Lazcano-Ponce E, Muñoz M, Huitrón G, Halley E, et al. Reference values for areal bone mineral density among a healthy Mexican population. Salud pública de México. 2009: 51 suppl 1: S56-S83.

21. Miraval Niño de Guzmán T, Segami SI, Chávez $\mathrm{CJ}$ et al. Fractura de cadera a trauma mínimo en mayores de 50 años: Morbimortalidad, pronóstico functional. Rev Per Reumatol. 2000; 6:68-5.

22. Becerra-Rojas F, Jupari M. Epidemiology of Osteoporosis in Peru. Bone. 2001; 29:294-313.

23. Aloia JF, McGowan D, Erens E, Miele G. Hip fracture patients have generalized osteopenia with a preferential deficit in the femur. Osteoporosis Int. 1992;2(2): 88-93. DOI: 10.1007/BF01623842

24. Morales-Torres J, Gutiérrez-Ureña S, Osteoporosis Committee of PANLAR The Burden of Osteoporosis in Latin America. Osteoporos Int. 2004; 15(8):625632. DOI: 10.1007/s00198-004-1596-3

25. Capunay MP, Vincentelo R, Dorregaray J, Angulo J. Incidencia de fractura de fémur proximal atribuible a osteoporosis en una población de Lima. J Clin Rheumatol 2002; 8: S49.

26. Ibáñez A, Rokes Ch, León G, Calvo A. Evaluación de la densidad mineral ósea radio en ultradistal en mujeres y hombres de la costa, sierra y selva del Perú. Rev. Peru. Reumatol. 2003; 9(1): 9-25.

27. Hosmer WD, Genant HK, Browner WS. Fractures before menopause: a red flag for physicians. Osteoporos Int. 2002; 13(4):337-41. DOI: 10.1007/ s001980200035

28. Sotelo W, Calvo A. Niveles de vitamina D en mujeres posmenopáusicas con osteoporosis primaria. Rev Médica Hered. 2011;22(1):10-14.

29. Melhus H, Snellman G, Gedeborg R, Byberg L, Berglund L, Mallmin $\mathrm{H}$, et al. Plasma 25-Hydroxyvitamin $\mathrm{D}$ levels and fracture risk in a communitybasedcohort of elderly men in Sweden. J Clin Endocrinol Metab. 2010; 95(6): 2637-2345. DOI: 10.1210/jc.2009-2699 